\title{
Efeito da orientação domiciliar no desempenho funcional de crianças com necessidades especiais
}

\author{
The effect of in-home instruction on the functional performance of \\ children with special needs
}

\author{
S.L. Pavão, F.P.S. Silva, N.A.C. Rocha
}

\begin{abstract}
Crianças com necessidades especiais apresentam disfunções neuro-sensório-motoras que limitam sua funcionalidade e participação social. Os cuidados dispensados pelos cuidadores a essas crianças podem reduzir essas limitações aumentando o grau de participação. O objetivo do presente estudo foi verificar o efeito da orientação domiciliar na funcionalidade de crianças com necessidades especiais em atividades de vida diária. Trinta e um cuidadores de crianças foram divididos aleatoriamente em dois grupos: Orientado (GO, $n=17$ ) e Controle (GC, $n=14$ ). Ambos os grupos foram submetidos ao PEDI (Pediatric Evaluation of Disability Inventory) no início do estudo e após três meses. Nesses três meses, o GO recebeu orientação domiciliar individualizada sobre banho, troca de roupas, alimentação e transporte da criança especial. A avaliação com o PEDI revelou aumento significativo nas pontuações do GO em auto-cuidado e função social na escala de habilidades funcionais, bem como em autocuidado na escala de assistência do cuidador. Sugere-se que a orientação domiciliar e individualizada a cuidadores seja eficaz para elevar o nível de funcionalidade de crianças com necessidades especiais em áreas de auto-cuidado e função social de acordo com o PEDI. Sendo, assim, uma forma simples e eficiente de intervenção junto à família.
\end{abstract}

Palavras-chave: orientação, cuidadores, desempenho funcional, crianças, PEDI

ABSTRACT

Children with special needs are known to have neurosensorymotor disorders that lead to both difficulties and limitations in their functionality and social participation. The care provided by caregivers to these children may reduce such limitations improving participation. The purpose of the present study was to verify the effect of in-home instruction on the functional performance of children with special needs during their daily activities. Thirty-one caregivers of children were randomly divided into two groups: Instructed (IG, $n=17$ ) and Control (CG, $n=14)$. Both groups were submitted to the Pediatric Evaluation of Disability Inventory (PEDI) at baseline and after three months. The IG received individualized in-home instruction over this three-month period on how to bathe, change clothes, feed and carry the child with special needs. The PEDI evaluation revealed that IG significantly increased PEDI score in the self-care and social function on the functional skills scale as well as in the self-care on the caregiver assistance scale. Individualized in-home instruction to caregivers is suggested to be effective in improving the level of independence of children with special needs in the self-care and social function in the PEDI. This is a simple and effective intervention with the family.

Keywords: instruction, caregivers, functional performance, children, PEDI

Submetido: 24.04.2010 | Aceite: 21.06.2010

Silvia Leticia Pavão, Fernanda Pereira dos Santos Silva e Nelci Adriana Cicuto Rocha. Universidade Federal de São Carlos, São Paulo, Brasil.

Endereço para correspondência: Silvia Leticia Pavão, Rua Rodrigues Alves, 731, CEP 17250-000, Centro Bariri, São Paulo, Brasil.

E-mail: silvia_pavao@hotmail.com 
As crianças que necessitam de cuidados especiais são aquelas que apresentam disfunções neuro-sensório-motoras congênitas ou adquiridas muito precocemente em seu desenvolvimento (Mutch, Alberman, Hagberg, Kodama, \& Perat, 1992; Schenker, Coster, \& Parush, 2005).

São crianças que apresentam prejuízos motores, comportamentais e de aprendizado, que limitam sua participação social no meio em que vivem e seu desempenho funcional na realização de atividades de vida diária (AVD) tornando-as pouco funcionais e dependentes de cuidados específicos (Beckung \& Hagberg, 2002; Farias \& Buchalla, 2005).

Este desempenho funcional é influenciado reciprocamente pelas características pessoais, intrínsecas da criança, pelas características do ambiente no qual elas se encontram (Tieman, Palisano, Gracely, \& Rosenbaum, 2004) e pelas demandas das tarefas propostas (Mancini et al., 2004) tais como alimentar-se, vestir-se, tomar banho e transportar-se (Sampaio et al., 2005; Schneidert, Hurst, Miller, \& Ustün, 2003).

A Classificação Internacional de Funcionalidade, Incapacidade e Saúde (CIF) desenvolvida pela Organização Mundial de Saúde (OMS), destaca a relação entre a deficiência apresentada pela criança e o ambiente em que ela se encontra, demonstrando que as características deste podem atuar tanto como facilitadoras, quanto como limitadoras da funcionalidade na criança (Dudgeon, Massagli, \& Ross, 1997; Farias \& Buchalla, 2005). Esta relação faz com que estas crianças tenham capacidades particulares em cada área do desenvolvimento (Hutton, Cooke, \& Pharoah, 1994), gerando uma demanda também particular no que se refere ao seu manuseio, ou seja, as formas mais adequadas de condução da criança em seu cuidado diário (Finnie, 2001).

Com base em tais aspectos, os programas de reabilitação devem direcionar seu protocolo de atendimento de acordo com as necessidades de cada criança, buscando seguir as etapas do desenvolvimento motor típico e trabalhando com desempenho em atividades funcionais (Hammal, Jarvis, \& Colver, 2004; Moura \& Silva, 2005).

Dentro desta abordagem, a orientação é um elemento de suporte familiar dos profissionais da saúde que eleva os níveis de percepção dos membros familiares em relação ao problema da criança (Kavalco, 2003; Motti, 2005).

A família tem um papel decisivo no processo de reabilitação infantil (Formiga, Pedrazzani, \& Tudella, 2004), especialmente nos casos de paralisia cerebral (Yano, 2003), uma vez que nos casos de super-proteção da criança, há um prejuízo no desenvolvimento de sua independência em habilidades funcionais (Lynch \& Hanson, 1992; Mancini et al., 2004).

Mancini et al. (2004), em seu estudo, verificaram o efeito do ambiente social no perfil funcional de crianças com paralisia cerebral, observando que a falta de estimulação por parte dos cuidadores pode fazer com que crianças com comprometimento motor moderado apresentem-se tão dependentes quanto crianças gravemente comprometidas.

Assim, o estudo demonstra que a intervenção familiar é importante, desde que bem direcionada. Kavalco (2003) também discute a importância da orientação familiar no dia-a-dia destas crianças, mas não a quantifica em relação a aspectos funcionais.

Desta maneira, embora se tenha amplamente discutido a importância da atuação familiar, há uma carência de estudos que verifiquem as influências de orientações direcionadas e domiciliares, aspecto esse que possibilita a identificação da real condição da família como contribuinte no processo de reabilitação.

Desta forma, o objetivo do presente estudo foi avaliar o efeito de um programa de orientação domiciliar, direcionado aos cuidadores de crianças com necessidades especiais, por meio da verificação do nível do desempenho funcional desta criança em atividades de vida diária antes e após o programa. 


\section{MÉTODO}

\section{Amostra}

Foram inicialmente selecionadas 50 crianças com necessidades especiais, divididas aleatoriamente em dois grupos: 25 no Grupo Orientado (GO) e 25 no Grupo Controle (GC). Dessas crianças, 15 não puderam ser contatadas e quatro foram descontinuadas ao longo do estudo por desmarcarem mais de três visitas seguidas. Portanto, restaram 31 crianças que fizeram parte do presente estudo, sendo 27 com diagnóstico médico de paralisia cerebral, duas de mielomeningocele, uma de síndrome de Down e uma de paralisia cerebral associada a síndrome de Down. Todas frequentavam um serviço de fisioterapia oferecido por duas instituições da cidade, e tinham idades entre 10 e 144 meses $(M=85.7, D P=45.2)$. Os participantes estavam divididos da seguinte maneira: 17 crianças e seus cuidadores no GO $(M=78.4$ meses, $D P=49.9)$ e 14 no GC $(M$ $=94.5$ meses, $D P=38.6)$.

As crianças eram dependentes na realização de uma ou todas as atividades de vida diária, tais como: alimentação, transporte, troca de roupas e banho; tinham classificação pelo GMFCS (Gross Motor Function Classification System; Palisano et al., 1997) de III $(n=7)$, IV $(n=10)$ ou $\mathrm{V}(n=14)$ e estavam em tratamento fisioterapêutico regular (duas vezes por semana) há pelo menos seis meses. Não foram selecionadas crianças residentes fora do município em que se realizou o estudo.

\section{Instrumentos}

Após assinarem o termo de consentimento livre e esclarecido, os cuidadores foram submetidos a um instrumento padronizado e validado de mensuração de habilidades funcionais da criança em atividades da rotina diária, o Pediatric Evaluation of Disability Inventory (PEDI; Mancini, 2005). O PEDI é dividido em três partes distintas que informam sobre três áreas do desempenho funcional. A primeira parte, com 197 itens avalia as habilidades funcionais da criança em atividades nas áreas de auto-cuidado (73 itens), mobilidade (59 itens) e função social (65 itens). A somatória dos escores resulta em um escore total bruto para cada uma das três áreas de habilidades funcionais. A segunda parte quantifica o auxílio fornecido pelo cuidador à criança em tarefas de auto-cuidado (8 itens), mobilidade (7 itens) e função social (5 itens). Nesta parte cada item é avaliado numa escala ordinal de 0 a 5 em que 0 indica necessidade de assistência máxima e 5 indica independência. A terceira parte do teste informa sobre as modificações necessárias para o desempenho das tarefas funcionais nas mesmas três áreas já descritas.

Para o presente estudo foram utilizadas apenas as duas primeiras partes do teste, referentes às áreas de habilidades funcionais e assistência do cuidador, com seus valores de escore bruto. Valores mais altos de escore indicam um melhor desempenho funcional da criança.

\section{Procedimentos}

$\mathrm{O}$ GO, além do tratamento fisioterapêutico convencional, participou de um programa de orientações composto por duas visitas domiciliares, com duração de aproximadamente uma hora cada visita. A primeira visita abordou os temas, banho e troca de roupas; e a segunda visita, alimentação e transporte adequado da criança. Em todas as visitas eram ensinadas $e$ demonstradas as formas corretas de posicionamento e estimulação da criança durante as atividades.

Para a realização do programa de orientações, a fisioterapeuta responsável passou por um processo de treinamento, de forma a padronizar e simplificar as informações transmitidas nas orientações. Foi também elaborada uma cartilha ilustrativa e sem termos técnicos, direcionada aos cuidadores, com instruções sobre o cuidado necessário para com a criança e formas adequadas de estimulação neuro-sensório-motora nas atividades de vida diária.

Durante cada orientação foi utilizado um kit com materiais úteis no cuidado diário da 
criança, e também com materiais para estimulação neuro-sensório-motora. Estes kits foram utilizados com o objetivo de ilustrar as intervenções propostas. O período decorrido entre cada um dos blocos de orientações (banho/troca de roupas e alimentação/ transporte) foi de 1 mês. A entrevista pós orientação (PO) ocorreu 3 meses ( \pm 7 dias) após a entrevista anterior à orientação domiciliar (AO).

$\mathrm{O}$ GC foi apenas submetido à entrevista $\mathrm{e}$ ao instrumento PEDI. A entrevista final ocorreu após um período de 3 meses $( \pm 7$ dias) da entrevista inicial. Neste intervalo de tempo as crianças obrigatoriamente deveriam estar submetidas a um programa de intervenção fisioterapêutica convencional.

A aplicação da entrevista e do PEDI foi realizada por duas fisioterapeutas experientes e treinadas no uso deste instrumento e que não tinham conhecimento dos procedimentos do estudo, uma realizou a avaliação inicial e a outra a final. O índice de concordância (interobservador) entre a entrevistadora da fase inicial e a da final foi de $80 \%$.

O estudo foi realizado pelo Laboratório de Análise do Desenvolvimento Infantil da Universidade Federal de São Carlos. Para que pudesse ser realizado, foi aprovado pelo Comitê de Ética em Pesquisa com Seres Humanos da Universidade Federal de São Carlos (Parecer n. ${ }^{\circ}$ 329-2007).

\section{Análise Estatística}

Para verificar a homogeneidade entre o GO e GC em relação ao nível de funcionalidade pela aplicação do instrumento PEDI e comprometimento da função motora grossa pelo GMFCS no início do estudo, antes de ser iniciado o programa de orientação, foi aplicado o teste de Mann-Whitney.

Os dados coletados na entrevista e no PEDI antes e após a intervenção foram analisados por meio de testes não-paramétricos, uma vez que são dados discretos. O teste utilizado para comparar dois grupos relacionados foi o teste de Wilcoxon. Para comparação dos grupos independentes foi utilizado o teste de MannWhitney. Foi adotado um nível de significância de $5 \%$. O software utilizado nas análises foi o SPSS 13.

\section{RESULTADOS}

O teste estatístico inicialmente aplicado demonstrou não haver diferença entre as crianças do GO e GC, antes do inicio da intervenção em relação aos escores da GMFCS $(U=92.5, p=.258)$ e aos escores do instrumento PEDI, tanto na área de habilidades funcionais, para os domínios de auto-cuidado $(U=110.5, p=.735)$, mobilidade $(U=96.0, p=.359)$ e função social $(U=109.5, p=.706)$, quanto na área de assistência do cuidador, para os domínios de auto-cuidado $(U=109.5, p=.697)$, mobilidade $(U=93.0, p=.273)$ e função social $(U=114.5, p=.857)$. Isto garante que as mudanças observadas ao final do estudo são decorrentes do processo de intervenção proposto. As mudanças observadas no instrumento PEDI para GO e GC antes e após o programa de orientação (AO e $\mathrm{PO}$ ) podem ser constatadas por meio das mudanças nos escores médios desses participantes, como mostra as Figuras 1 e 2.

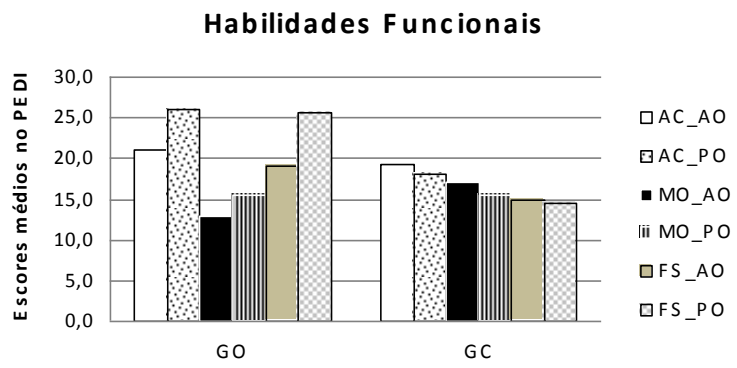

Figura 1. Mudanças observadas nos escores médios do PEDI para grupo orientado (GO) e grupo controle (GC) na área de habilidades funcionais, nos domínios de auto-cuidado (AC), mobilidade (MO) e função social (FS), antes da orientação (AO) e pós orientação (PO)

$\mathrm{Na}$ análise da pontuação PEDI no domínio de habilidades funcionais para a área de autocuidado, constatou-se uma melhora significativa dos escores dos integrantes do GO 
( $Z=-3.41, p<.001)$, enquanto que no GC, essa melhora não ocorreu, demonstrando que a orientação aumenta a capacidade de participação da criança em seus próprios cuidados. $\mathrm{Na}$ área de mobilidade observou-se para o GO um ganho também significativo nos escores dos participantes $(Z=-2.40, p=$ .014 ), ao passo que para o GC os ganhos não tiveram relevância estatística. $\mathrm{Na}$ área de função social foi também observada uma diferença estatisticamente significativa entre os grupos. Verificou-se que os integrantes do GO apresentaram ganhos nos escores desta área $(Z$ $=-3.41, p<.001)$. No GC o mesmo não ocorreu.

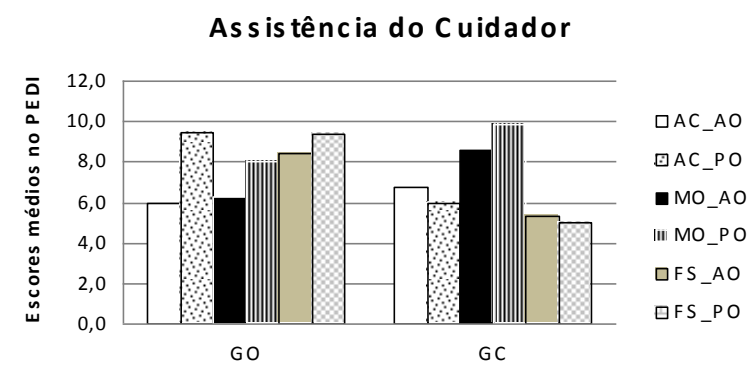

Figura 2. Mudanças observadas nos escores médios do PEDI para grupo orientado (GO) e grupo controle (GC) na área de assistência do cuidador, nos domínios de auto-cuidado (AC), mobilidade (MO) e função social (FS), antes da orientação (AO) e pós orientação (PO)

A análise da pontuação do PEDI no domínio assistência do cuidador revelou um aumento significativo nos escores na área do autocuidado para o GO $(Z=-2.77, p=.004)$. Para o GC não houve diferença significativa $(Z=$ $-.56, p=.633$ ), o que indica o sucesso da orientação para minimizar a necessidade de assistência no auto-cuidado. Nas áreas de mobilidade e função social neste domínio não houve diferenças estatisticamente significativas nos escores para ambos os grupos nas duas áreas $(Z=-1.07, p>.05$ e $Z=-1.89, p>$ .05 , respectivamente).

\section{DISCUSSÃO}

Os resultados do presente estudo fornecem subsídios de que o programa de orientação domiciliar exerceu influência no desempenho funcional de crianças com necessidades especiais, de acordo com a avaliação realizada por meio do instrumento PEDI. A realização da orientação aos cuidadores, em sua maioria mães, pode ter possibilitado o fortalecimento da díade pais-criança no seu domicílio, facilitando a inclusão da criança nas atividades familiares. Estudos apontam que quando devidamente instruídos, os pais não só viabilizam esta sociabilização, como também ampliam o nível de independência da criança (Hostler, 1999; Leite \& Prado, 2004; Lindeke, Leonard, Presler, \& Garwick, 2002; Martinez, Joaquim, Oliveira, \& Santos, 2007).

A atuação dos cuidadores, quando inadequada, apenas desestabiliza o processo de desenvolvimento da criança e pode comprometer seu adequado tratamento (Lopes, Kato, \& Corrêa, 2002; Mancini et al., 2004; Mendes, Nunes, \& Ferreira, 2002). Assim, ressalta-se a relevância de um programa de orientação, pois quando bem orientado o cuidador pode direcionar adequadamente o cuidado que dispensa à criança e contribuir com o processo de reabilitação em casa, local em que passa a maior parte do tempo (Leite \& Prado, 2004).

Pôde-se observar que o GO apresentou uma melhora no domínio de habilidades funcionais na área de auto-cuidado da PEDI, indicando que as crianças passaram a ter uma participação mais expressiva em atividades como troca de roupas, higiene pessoal e alimentação, o que parece estar relacionado a um aumento da independência nas AVD. Essa melhoria tem relevância clínica uma vez que as crianças envolvidas no estudo apresentavam comprometimento motor de moderado a severo (níveis III, IV e V no GMFCS).

É conhecido que a função motora grossa está diretamente relacionada com o nível de funcionalidade apresentado pela criança (Mancini et al., 2002); e quanto maior o comprometimento desta função, maior será a dificuldade na aquisição de habilidades funcionais (Haley, Coster, Ludlow, 
Haltiwanger, \& Andrellos, 1992; Lepage, Noreau, \& Bernard, 1998). Segundo Ostensjø, Carlberg e Vøllestad (2005), o aumento no nível de GMFCS é inversamente proporcional à pontuação atingida pela criança no PEDI. Assim, ressalta-se o efeito positivo do programa de orientação domiciliar dada a dificuldade de crianças tão severamente comprometidas adquirirem habilidades funcionais.

Resultados positivos podem ser atribuídos ao direcionamento do programa para a prática de atividades funcionais com a criança em AVDs, pela sua realização domiciliar e pela individualidade das orientações. Esse conjunto de fatores permite que as propostas de adequações apresentadas aos cuidadores sejam pertinentes e cabíveis ao seu contexto ambiental e socioeconômico, de maneira que pudessem ser efetivamente realizadas e sanadas as potenciais dúvidas dos cuidadores.

Autores defendem a importância da busca de intervenções para aumentar o grau de independência da criança, na tentativa de atenuar as restrições funcionais que ela encontra diante da sociedade (Beckung \& Hagberg, 2002; Hammal et al., 2004; Kavalco, 2003; Ostensjø, Carlberg, \& Vøllestad, 2003). Mancini et al. (2002) acreditam que o bom desempenho das crianças em habilidades funcionais é um objetivo importante no processo de reabilitação, uma vez que lesões no sistema nervoso central (SNC) limitam a realização de atividades e tarefas do cotidiano, especialmente na área do auto-cuidado. Da mesma forma, o uso de atividades funcionais é preconizado para que se tenha um aumento do grau de independência da criança (Ketelaar, Vermeer, Hart, van Petegem-van Beek, \& Helders, 2001).

No domínio de assistência do cuidador, ainda na área de auto-cuidado, constatou-se menor necessidade do auxílio às crianças, o que pode representar um aumento em sua independência. Isso ressalta que a instrução dos cuidadores torna mais adequado o auxílio por eles prestado à criança nos cuidados diários, permitindo que ela tenha uma participação mais ativa em suas próprias atividades (Colnago \& Biasóli-Alves, 2003; Formiga et al., 2004; Lopes et al., 2002; Mendes et al., 2002).

$\mathrm{Na}$ área da mobilidade, foi verificada diferença estatisticamente significativa apenas para o domínio de habilidades funcionais. No domínio de assistência do cuidador não foram observadas diferenças significativas entre os grupos. Tais resultados indicam que embora as crianças tenham adquirido habilidades de mobilidade com o programa de orientações, permaneceram com o mesmo grau de dependência em relação a seus cuidadores, necessitando de auxílio para locomover-se no ambiente interno, mesmo em curtas distâncias. Segundo Palisano et al. (2003), a mobilidade é uma área que envolve funções complexas e difíceis de serem alcançadas em crianças com comprometimento motor tão severo (níveis III, IV e V do GMFCS). Desta forma, acredita-se que somente a orientação não foi o suficiente para mudar de forma expressiva as necessidades de assistência da criança nesta área e que o pequeno aumento do escore na mobilidade, observado em ambos os grupos, seja devido ao tratamento fisioterapêutico regular pelo qual todas as crianças do estudo foram submetidas. Assim vale ressaltar que, para que mudanças significativas ocorram nesta área, além da orientação domiciliar, é também necessário um incremento no programa de reabilitação, e até fazer uso de equipamentos adaptativos.

Farias e Buchalla (2005) e Palisano et al. (1997) defendem o adequado conhecimento sobre adaptações, para que o ambiente possa atuar como um facilitador da funcionalidade, especialmente no caso da locomoção. No presente estudo, verificou-se que o problema maior não foi o conhecimento, pois esse estava sendo oferecido aos cuidadores, mas sim as condições de moradia, que podem ter restringido muito mais do que promovido a função de locomoção. Portanto, uma limitação do estudo pode ter sido o não cumprimento 
dos cuidadores das orientações realizadas devido às condições físicas das casas visitadas, em sua maioria pequenas e com barreiras arquitetônicas para a mobilidade da criança. Isto pode ter restringido essa função da criança, justificando a dependência de assistência, mesmo após o programa de orientações.

$\mathrm{Na}$ área da função social foram constatadas melhoras no domínio de habilidade funcionais, indicando que as crianças do GO possivelmente passaram a apresentar maior participação na sociedade, nas atividades familiares e na interação com outras crianças em eventos sociais. Acredita-se que o ganho nesta área deveu-se ao trabalho desenvolvido com os cuidadores, visando a promoção da adequada estimulação dos sentidos das crianças, a promoção do diálogo durante as atividades de vida diárias desenvolvidas com elas e a sua inserção nas atividades de rotina e lazer da família.

Quanto à função social da criança no domínio de assistência do cuidador, não se observou melhoras após a orientação. Isso indica que, mesmo pós orientações, as crianças continuaram recebendo assistência para desempenhar suas funções sociais. Desta forma, a avaliação dos domínios de habilidades funcionais e de assistência do cuidador na área da função social pode ter apresentado resultados diferentes pelo fato de que, embora a criança tenha melhorado suas habilidades funcionais na área da função social para interagir melhor com o meio em que está inserida, há casos em que a postura do cuidador é de tamanho zelo que a assistência prestada ainda é intensa, limitando a independência da criança em suas relações sociais. Segundo Lynch e Hanson (1992), os pais brasileiros de crianças especiais têm uma postura histórica de super-proteção que acaba por mascarar as habilidades da criança e prejudicar seu desenvolvimento motor. Junto disso, a função social é um conjunto complexo que além de sofrer influências do desempenho motor, também tem relação com a idade da criança e problemas de aprendizado, sendo, portanto, uma área de poucos ganhos observáveis na presença de necessidades especiais (Ostensjø, Carlberg, \& Vøllestad, 2004). Assim, embora a melhora observada nesta área tenha se restringido ao domínio de habilidades funcionais, ela tem grande expressividade dada a dificuldade de ganhos nesta área para estas crianças.

Foi possível, desta forma, identificar dois pontos importantes. O primeiro, que a orientação aos cuidadores realizada em domicílio é uma etapa fundamental no planejamento do tratamento, possibilitando contemplar na reabilitação, mudanças na estrutura e função do corpo e otimização das capacidades funcionais e sociais da criança, como preconizada pela OMS (Farias \& Buchalla, 2005). O segundo ponto, que a orientação aos cuidadores reforça a relação entre a deficiência apresentada pela criança e o ambiente em que ela se encontra, podendo esse ambiente atuar como facilitador da funcionalidade (Dudgeon et al., 1997; Farias \& Buchalla, 2005).

A orientação dos cuidadores de forma individual e no ambiente domiciliar tem um efeito direto no aumento do desempenho funcional da criança com necessidades especiais em atividades de vida diárias nas áreas de auto-cuidado, mobilidade e função social. Sendo, assim, uma forma simples e eficiente de intervenção terapêutica junto à família. No entanto, entende-se que o estudo contou com uma amostra relativamente pequena e pouco homogênea em sua composição, fatores que foram limitações do presente estudo.

\section{REFERÊNCIAS}

Beckung, E., \& Hagberg, G. (2002). Neuroimpairments, activity limitations, and participation restrictions in children with cerebral palsy. Developmental Medicine Child Neurology, 44(5), 309-316.

Colnago, N. A., \& Biasóli-Alves, Z. M. (2003). Necessidades de famílias de bebês com Síndrome de Down: Subsídios para uma 
proposta de intervenção. In M. C. Marchezini, M. A. Almeida, E. D. Tanaka, \& S. Omote (Eds.), O papel da família junto ao portador de necessidades especiais (pp. 169-180). Londrina: EDUEL.

Dudgeon, B. J., Massagli, T. L., \& Ross, B. W. (1997). Educational participation of children with spinal cord injury. American Journal of Occupational Therapy, 51(7), 553-561.

Farias, N., \& Buchalla, C. M. (2005). A classificação internacional de funcionalidade, incapacidade e saúde da organização mundial da saúde: Conceitos, usos e perspectivas. Revista Brasileira de Epidemiologia, 8(2), 187-193.

Finnie, N. A. (2001). O manuseio em casa da criança com paralisia cerebral ( $3^{\mathrm{a}}$ ed.). São Paulo: Manole.

Formiga, C. K. M., Pedrazzani, E. S., \& Tudella, E. (2004). Desenvolvimento motor de lactentes pré-termo participantes de um programa de intervenção fisioterapêutica precoce. Revista Brasileira de Fisioterapia, 8(3), 239-245.

Haley, S. M., Coster, W. J., Ludlow, L. H., Haltiwanger, J. T., \& Andrellos, P. J. (1992). Pediatric Evaluation of Disability Inventory: Development, standardization, and administration manual (v. 1.0). Boston, MA: Trustees of Boston University, Health and Disability Research Institute.

Hammal, D., Jarvis, S. N., \& Colver, A. F. (2004). Participation of children with cerebral palsy is influenced by where they live. Developmental Medicine Child Neurology, 46(5), 292-298.

Hostler, S. L. (1999). Pediatric family-centered rehabilitation. Journal of Head Trauma Rehabilitation, 14(4), 384-393.

Hutton, J. L, Cooke, T., \& Pharoah, P. O. (1994). Life expectancy in children with cerebral palsy. British Medical Journal, 309(6952), 431-435.

Kavalco, T. F. (2003). A eficácia de orientações de posicionamentos sentados funcionais aplicadas no domicílio para familiares de uma criança portadora de paralisia cerebral: Um estudo de caso. Monografia não publicada, Universidade Estadual do Oeste do Paraná, Paraná, Brasil.

Ketelaar, M., Vermeer, A., Hart, H., van Petegemvan Beek, E., \& Helders, P. J. (2001). Effects of a functional therapy program on motor abilities of children with cerebral palsy. Physical Therapy, 81 (9), 1534-1545.

Leite, J. M. R., \& Prado, G. F. (2004). Paralisia cerebral: Aspectos fisioterapêuticos e clínicos. Revista Neurociências, 12, 41-45.
Lepage, C., Noreau, L., \& Bernard, P. M. (1998) Association between characteristics of locomotion and accomplishment of life habits in children with cerebral palsy. Physical Therapy, 78(5), 458-469.

Lopes, G. B., Kato, L. S., \& Corrêa, P. R. C. (2002). Os pais das crianças com deficiências: Reflexões acerca da orientação em reabilitação motora. Psicologia: Teoria e Prática, 4(2), 67-72.

Lynch, E., \& Hanson, M. J. (1992). Developing crosscultural competence: A guide for working with children and their families. Baltimore: Paul $\mathrm{H}$. Brookes.

Lindeke, L. L., Leonard, B. J., Presler, B., \& Garwick, A. (2002). Family-centered care coordination for children with special needs across multiple settings. Journal of Pediatric Health Care, 16(6), 290-297.

Mancini, M. C., Fiúza, P. M., Rebelo, J. M., Magalhães, L. C., Coelho, Z. A. C., \& Paixão, M. L. (2002). Comparação do desempenho de atividades funcionais em crianças com desenvolvimento normal e crianças com paralisia cerebral. Arquivos de Neuro-Psiquiatria, 60(2B), 446-452.

Mancini, M. C., Alves, A. C. M., Schaper, C., Figueiredo, E. M., Sampaio, R. F., Coelho, Z. A., et al. (2004). Gravidade da paralisia cerebral e desempenho funcional. Revista Brasileira de Fisioterapia, 8(3), 253-260.

Mancini, M. C. (2005). Inventário de Avaliação Pediátrica de Incapacidade (PEDI): Manual da versão brasileira adaptada. Belo Horizonte: Editora UFMG

Martinez, C. M. S., Joaquim, R. H. V., Oliveira, E. B., \& Santos, I. C. (2007). Suporte informacional como elemento para orientação de pais de pré-termo: Um guia para o serviço de acompanhamento do desenvolvimento no primeiro anos de vida. Revista Brasileira de Fisioterapia, 11 (1), 73-81.

Mendes, E. G., Nunes, L. R., \& Ferreira, J. R. (2002). Atitudes e percepções acerca dos indivíduos com necessidades educacionais especiais. Temas em Psicologia, 10, 121-134.

Motti, T. M. F. (2005). Programa da orientação não presencial de pais de crianças deficientes auditivas. Tese de doutoramento, Universidade Federal de São Carlos, São Paulo, Brasil.

Moura, E. W., \& Silva, P. A. C. (2005). Fisioterapia: Aspectos clínicos e práticos da reabilitação. São Paulo: Artes Médicas. 
Mutch, L., Alberman, E., Hagberg, B., Kodama, K., \& Perat, M. V. (1992). Cerebral palsy epidemiology: Where are we now and where are we going? Developmental Medicine Child Neurology, 34(6), 547-551.

Ostensjø, S., Carlberg, E. B., \& Vøllestad, N. K. (2003). Everyday functioning in young children with cerebral palsy: Functional skills, caregiver assistance, and modifications of the environment. Developmental Medicine Child Neurology, 45(9), 603-612.

Ostensjø, S., Carlberg, E. B., \& Vøllestad, N. K. (2004). Motor impairments in young children with cerebral palsy: Relationship to gross motor function and everyday activities. Developmental Medicine Child Neurology, 46(9), 580-589.

Ostensjø, S., Carlberg, E. B., \& Vøllestad, N. K. (2005). The use and impact of assistive devices and other environmental modifications on everyday activities and care in young children with cerebral palsy. Disability \& Rehabilitation, 27(14), 849-861.

Palisano, R. J., Rosenbaum, P., Walter, S., Russell, D., Wood, E., \& Galuppi, B. (1997). Development and reliability of a system to classify gross motor function in children with cerebral palsy. Developmental Medicine Child Neurology, 39(4), 214-223.

Palisano, R. J., Tieman, B. L., Walter, S. D., Bartlett, D. J., Rosenbaum, P. L., \& Russell, D. (2003). Effect of environmental setting on mobility methods of children with cerebral palsy. Developmental Medicine Child Neurology, 45(2), 113-120.

Sampaio, R. F., Mancini, M. C., Gonçalves, G. G. P., Bittencourt, N. F. N., Miranda, A. D., \& Fonseca, S. T. (2005). Aplicação da classificação internacional de funcionalidade, incapacidade e saúde (CIF) na prática clínica do fisioterapeuta. Revista Brasileira de Fisioterapia, 9(2), 129-136.

Schenker, R., Coster, W., \& Parush, S. (2005). Participation and activity performance of students with cerebral palsy within the school environment. Disability \& Rehabilitation, 27(10), 539-552.

Schneidert, M., Hurst, R., Miller, J., \& Ustün, B. (2003). The role of environment in the International Classification of Functioning, Disability and Health (ICF). Disability $\mathcal{E}$ Rehabilitation, 25(11/12), 588-595.

Tieman, B. L., Palisano, R. J., Gracely, E. J., \& Rosenbaum, P. L. (2004). Gross motor capability and performance of mobility in children with cerebral palsy: A comparison across home, school, and outdoors/community settings. Physical Therapy, 84(5), 419-429.

Yano, A. M. M. (2003). As práticas de educação em famílias de crianças com paralisia cerebral diplégica espástica e com desenvolvimento típico pertencentes a camadas populares da cidade de Salvador. Tese de doutoramento, Universidade de São Paulo, São Paulo, Brasil.

(c) EY-No Todo o conteúdo da revista Motricidade está licenciado sob a Creative Commons, excepto quando especificado em contrário e nos conteúdos retirados de outras fontes bibliográficas. 